\title{
Dental considerations in a patient
}

with haemophilia

Chandra Khyati, Mavinakote Gowda Triveni, Rini Gopal, AB Tarunkumar, Suresh Hanagavadi, Dhoom Singh Mehta

Haemophilia is a rare blood clotting disorder, characteristic features of which include extemporaneous and post-traumatic subcutaneous bleeding and mucosal haemorrhages. Genetic deficiency of coagulation factor VIII results in haemophilia $A$, while deficiency of factor IX leads to haemophilia $B$. The most common treatment for haemophilia $A$ is administration of recombinant or plasma-derived factor VIII concentrate, to raise the levels of the deficient factor VIII. Tranexamic acid is also used as an anti-fibrinolytic agent that inhibits plasminogen activators present in oral secretion and stabilises the clot. Administration of factor IX is required in haemophilia B. Treatment leads to increased longevity and quality of life for patients. Dental conditions and treatments are more complicated and uncertain in patients with haemophilia due to bleeding risk, thus restorative dental care is of paramount importance for those with haemophilia. The fear of bleeding during treatment procedures is the primary cause of lack of proper dental care for people with haemophilia in countries with limited health care resources. This case report highlights the significance of clinical examination and investigation, and the importance of proper interaction between a haematologist and the periodontist for correct multidisciplinary and uneventful management of periodontal health of a patient with haemophilia.

Keywords: haemophilia, spontaneous bleeding, factor VIII, tranexamic acid.

The genetic deficiency of clotting factor VIII causes haemophilia $A$, an inherited $X$-linked recessive trait found in males. The prevalence of haemophilia $A$ is approximately 1 in 5,000 males [1]. Dysfunctional factor VIII protein is the causative factor for haemophilia A in $5-10 \%$ of patients, with 90-95\% affected due to quantitative deficiency. Familial

Dr Chandra Khyati, postgraduate student, Department of Periodontics, Bapuji Dental College and Hospital, Davangere, Karnatka, India Dr Mavinakote Gowda Triveni, Professor, Department of Periodontics, Bapuji Dental College and Hospital, Davangere, Karnatka, India. Email: 2012mgtriveni@gmail.com

Dr AB Tarunkumar, Professor, Department of Periodontics, Bapuji Dental College and Hospital, Davangere, Karnatka, India

Dr Rini Gopal, Postgraduate student, Department of periodontics, Bapuji

Dental College and Hospital, Davangere, Karnatka, India

Dr Suresh Hanagavadi, Professor, Department of Pathology and

Haematology, JJM Medical College, Davangere, Karnataka, India

Dr Dhoom Singh Mehta, Professor and Head, Department of Periodontics,

Bapuji Dental College and Hospital, Davangere, Karnatka, India

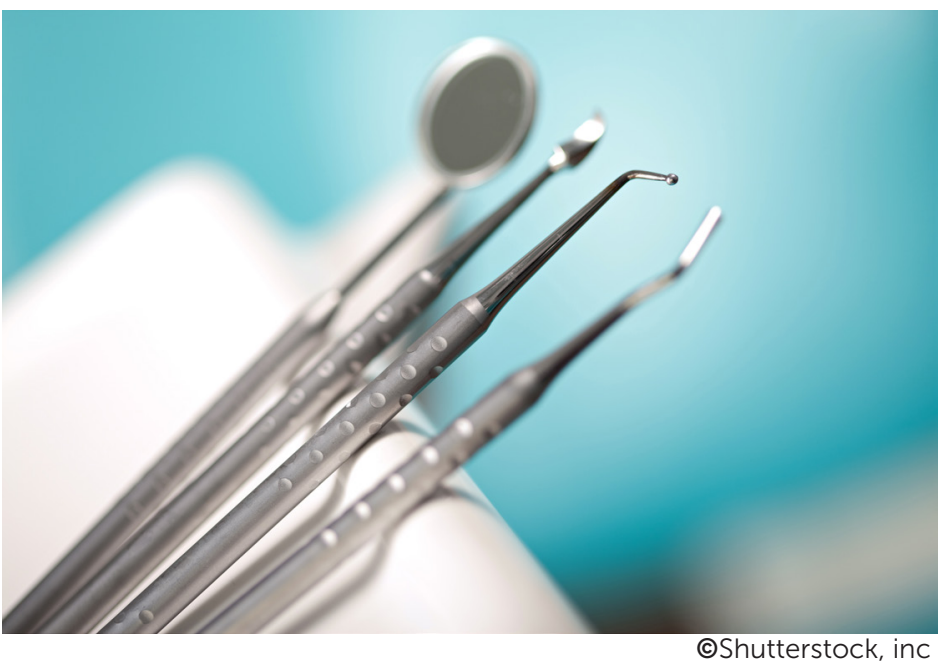

inheritance is not found in approximately $30 \%$ of patients, where the apparent cause is a new mutation. Symptoms include delayed bleeding, ecchymosis, deep haematomas, epistaxis, spontaneous gingival bleeding and haemarthrosis. The normal range for factor VIII is 50-150\% (50-150 IU/dl). According to World Federation of Hemophilia guidelines, the amount of the clotting factor in the blood determines the severity of haemophilia [1]. A factor VIII level of $5-40 \%$ (5-40 lU/dl or $0.05-0.40 \mathrm{lU} / \mathrm{ml})$ of normal factor activity (mild haemophilia) is associated with bleeding during trauma or some surgery, $1-5 \%(1-5 \mathrm{lU} / \mathrm{dl}$ or $0.01-0.05 \mathrm{lU} /$ $\mathrm{ml}$ ) of normal factor activity (moderate haemophilia) shows bleeding after mild injury; $<1 \%(<1 \mathrm{lU} / \mathrm{dl}$ or $0.01 \mathrm{lU} / \mathrm{ml})$ of normal factor activity (severe haemophilia) is associated with spontaneous bleeding [2]. Intravenous infusion of the deficient clotting factors controls or prevents bleeding. Some patients with mild or moderate forms of haemophilia $A$ are treated with desmopressin (DDAVP) to achieve transient increase in factor VIII levels. The highly vascular oral cavity is the hotspot for haemorrhage in people with haemophilia, in whom correct dental treatment is of paramount importance $[1,3]$.

Haemophilia A and B are inherited bleeding disorders, caused due to low factor concentrations of factor VIII and IX respectively. Impaired secondary haemostasis is responsible for the bleeding. Presentation of post-bleeding, or previously identified and known familial history aid the diagnosis of haemophilia A and B. There is no significant difference in the clinical presentation of haemophilia $A$ and $B$ and they are clinically not distinguishable. A specific factor 

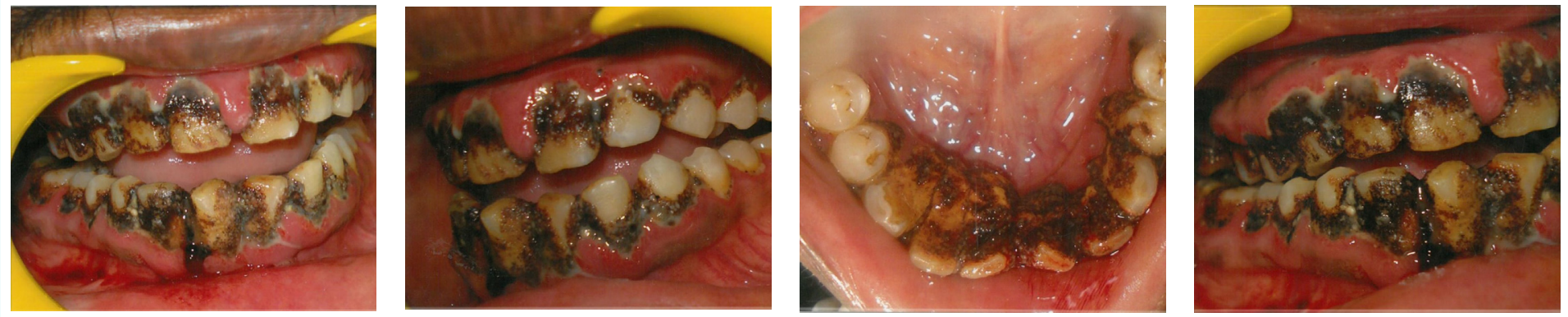

Figure 1: Preoperative intraoral view of the patient (frontal view, left buccal view, lingual view and right buccal view)

assay therefore plays an important role in confirmation of the diagnosis. Initial haemostasis tests included activated partial thromboplastin time (aPTT), and prothrombin time (PT), all of which are mandatory if the preliminary clinical assessment advocates a bleeding disorder [4]. Specific factor assays are necessary in case of a bleeding history.

The main focus of treatment for inherited bleeding disorders is to raise the concentration of the lacking factor adequately in order to arrest bleeding, as seen in severe cases and trauma or during an elective surgery [4]. Desmopressin (an analogue of antidiuretic hormone vasopressin) is an effective, and cost-effective, treatment modality for mild haemophilia A patients. Furthermore, adjuvant and adjunctive therapies, including fibrin glue and antifibrinolytic amino acids (tranexamic acid) may also be used. Factor IX concentrates are used in the treatment of patients with haemophilia B. For patients diagnosed with an inhibitor, immune tolerance regimens have been derived using porcine and human factor VIII as a toleragen. These studies have shown favourable results, and this treatment should be considered for newly diagnosed patients with inhibitors $[13,14]$. The therapy, designed to overwhelm the immune system with high doses of factor VIII, attempts to attain immune tolerance in a manner analogous to treating allergies, and was first introduced in 1974 by Brackmann [5]. The best results are obtained when therapy is initiated without recent exposure to factor VIII, or in patients with peak levels of $100 \mathrm{BU}$, with recent inhibitor development. Patients receive continued prophylactic doses of factor to overpower inhibitor development [5]. Depending on the regimen used, it is noteworthy that the disappearance of the inhibitor occurred earlier, within 15 months. This means that treatment must be continued for a considerably longer time after the inhibitor is not detectable and that if the inhibitor persists after two years of treatment, the probability of a success becomes virtually nil. Tolerance was shown to be a stable condition with relapses rarely occurring [15]. The high-dose regimen involves the usage of immunosuppressants.

Astudy showed that the fluid from inflamed gingival crevices possesses more plasmin activity and the reaction to FDPL (Fibrin Degradation products Latex Test) in comparison to the healthy gingiva [6]. It is therefore imperative to maintain the gingival health in haemophilic patients.

\section{Case report}

A 30-year-old man reported to the department of periodontics at Bapuji Dental College and Hospital Davangere, India, complaining of bleeding gums, which had persisted for the past 8-10 days. He had no past dental history, had not undergone any dental procedure in the past and had a reasonably good oral health status in terms of gingival and periodontal health. But the medical history indicated that he was known to have haemophilia since birth with a significant family history. Intraoral examination revealed spontaneous gingival bleeding (Figure 1). The patient was very well educated and aware with regard to haemophilia and its complications, thus treatment planning and protocol was of not an issue. He was sent for routine blood investigation, which revealed a prolonged activated partial thromboplastin time (APTT), normal PT and normal haemoglobin. A factor assay was undertaken, which confirmed the patient's severe haemophila A. This meant that it was imperative to replace factor VIII by infusion before any invasive dental procedure, due to the possibility of bleeding. Prophylactic antibiotic coverage of amoxicillin was prescribed. After consultation, the patient was advised to undergo factor infusion one hour before the procedure (scaling and root planing). Prior to the dental prophylaxis in order to prevent any bleeding episodes, and given that the patient had severe haemophilia, he was managed according to a recognised protocol [7]. Tranexamic acid 10 $\mathrm{mg} / \mathrm{kg}$ in $20 \mathrm{ml}$ normal saline was infused preoperatively over 20 minutes (with $1 \mathrm{~g}$ every 8 hours orally for 5 days postoperatively) with intravenous factor VIII replacement to build up to $50-75 \%$ along with local haemostatic measures and tranexamic acid mouth rinse and factor VIII maintenance for 7 days and postoperative care. The dose to be infused to raise the factor VIII concentrate was calculated by using the formula: Dose to be infused (units) $=\{$ weight $(\mathrm{kg}) *$ increment needed $(\mathrm{IU} / \mathrm{dl})\} / 2$ [7]. The patient weighed $70 \mathrm{~kg}$ and so $2800 \mathrm{lU} / \mathrm{dl}$ was infused one hour before the procedure in order to raise his factor VIII level by $80 \%$. After 10 hours, $1400 \mathrm{lU} / \mathrm{dl}$ was infused for next 3 days and tapered to $700 \mathrm{lU} / \mathrm{dl}$ for another 3 days.

Local measures are important in minimising the risk of post-operative bleeding; thus, even a minor procedure, such as dental procedures, should be done with minimum trauma to soft tissues. In this case, ultrasonic scaling was preferred over hand scaling. Initial factor VIII replacement allowed clots to form, and tranexamic acid permitted them 

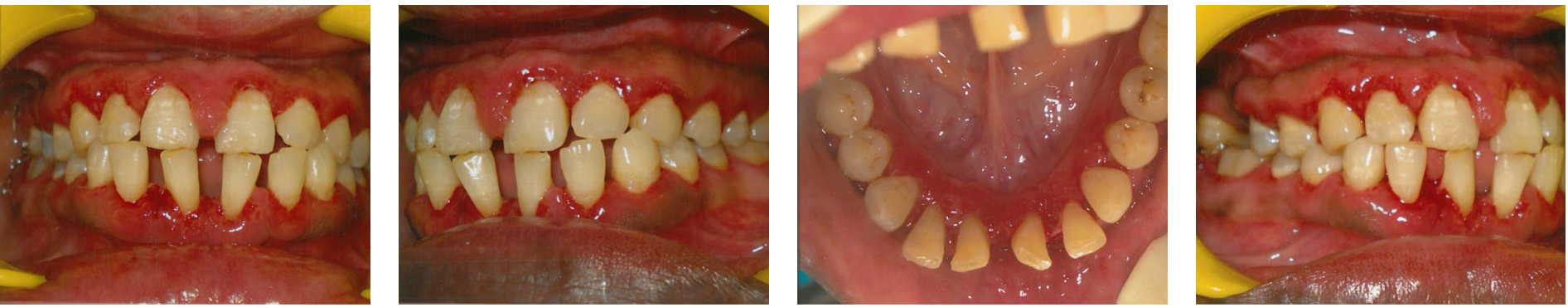

Figure 2: Postoperative inraoral view of the patient (frontal view, left buccal view, lingual view and right buccal view)

to remain haemostatically effective. Thus, the bleeding was controlled and the dental care was completed in single sitting (Figure 2). According to the European Haemophilia Standardisation Board Survey, antifibrinolytic therapy is normally administered for 5-10 days [8]. The patient was therefore advised to use tranexamic acid mouthwash with $10 \mathrm{ml}$ of $4.8-5 \%$ solution $4-5$ times a day for 2 minutes [5]. The rinsing was advised to be carried out for 2-5 days and was extended to 8 days. The patient was kept under observation and a factor VIII assay was done post-operatively in order to check factor levels and plan factor supplementation. The patient was successfully returned back to the proper oral hygiene status by following the proper principles of haemostasis, careful management of soft tissues and proper communication between the haematologist and periodontist during the treatment period.

\section{Discussion}

Characteristic symptoms of haemophilia include external or internal bleeding episodes. The disorder leads to a severely increased risk of prolonged bleeding from common injuries, or bleeds may be spontaneous and without obvious cause. Bleeding may occur anywhere in the body. Muscle and joint haemorrhages are the most serious symptoms. Where dental care is concerned, interaction between the haematologist and dentist is essential to management, with or without the presence of trauma [5]. Bleeding disorders are a major concern for dental treatment as saliva in the oral cavity has high amount of tissue plasminogen activator, which helps in activating plasmin, an enzyme that is active in the breakdown of fibrin in clots. In order to stabilise the clot for longer period of time, antifibrinolytic agents such as tranexamic acid, can be used in combination with factor therapy in severe haemophilia cases; this is sometimes the only therapy needed in mild to moderate haemophilia patients [9]. Local measures to control haemorrhage typically take one of three forms: haemostatic pads, antifibrinolytics or topical thrombin. Topical thrombin is useful and effective in some clinical situations, but as it must come into direct contact with the bleeding site, it is advised to be used directly under pressure. Topical thrombin is ineffective when used with oxidised cellulose due to its acidic nature. To form a haemostatic patch, thrombin and cryoprecipitate can be used togather. In addition to tranexamic acid, postoperative use of Epsilon Amino Caproic Acid significantly reduces the amount of factor needed to control bleeding when used alongside presurgical infusion of factor.

Periodontal health is of critical importance for the haemophiliac for two principle reasons [6]:

- Hyperaemic gingiva contribute to spontaneous and induced gingival bleeding.

- Periodontitis is a leading cause of tooth morbidity, necessitating extraction.

There are certain points that a dentist needs to be aware of while treating people with haemophilia, including the patient's factor level, previous bleeding episodes, what kind of factor product is being administered in treatment, and the frequency of infusions per year. This information is valuable not only in normal treatment, but also for those patients who are not responsive to routine therapy (i.e. those who develop inhibitors).

In periodontal surgery, minimal trauma to soft tissues and bone and postoperative hygiene is essential. Suturing is essential to stabilise gum flaps; a non-traumatic needle must be used and number of sutures should be minimised. Vicryl sutures are preferred and catgut is best avoided [7]. When the patient is responsive to the factor replacement therapy, factor concentrate is infused one hour prior to the procedure to raise the level to $30 \%$ in moderate cases [10]. In the case described here, the patient had a severe factor deficiency so the factor level was raised to $80 \%$. Currently, in addition to ultrasonic scaling, the use of laser assisted scaling and root planing can help in avoiding excessive bleeding in haemophilic patients during the procedure, and diminish the problem of aerosol production with ultrasonic scaling. Electrocautery and the use of chair-side lasers can assist in the prevention of bleeding that might arise postperiodontal therapy.

The basis of treatment is to raise the concentration of the missing factor sufficiently to prevent spontaneous and traumatic bleedings or to cover surgery. This can be done by the administration of concentrates of the missing factor. Certain commonly prescribed drugs in dentistry interfere with haemostasis, including non-steroidal anti-inflammatory drugs (NSAIDs), some antibiotics and anti-fungals and certain other medications that include omeprazole, phenytoin, corticosteroids, carbamazipine, barbiturates [12]. Some local techniques require haemostatic coverage, such as inferior dental block and 
lingual infiltration; those that do not require haemostatic coverage include buccal infiltration, intra-papillary and intra-ligamentary injections [11].

In the case discussed here, effective periodontal treatment was provided with no postoperative bleeding episodes and complications, as a result of taking into consideration the principles and contraindications relevant in treating him. Thus, it is possible to provide haemophiliac patients with appropriate dental care and uneventful dental treatment by multidisciplinary team management, and timely and proper communication between the dentist and haematologist.

\section{Disclosures}

The authors have advised that there are no interests that might be perceived as posing a conflict or bias.

This is an Open Access article distributed under the terms of the Creative Commons Attribution License (http://creativecommons.org/licenses/ by/2.0), which permits unrestricted use, distribution, and reproduction in any medium, provided the original work is properly cited.

\section{References}

1. Zaliuniene R, Peciuliene V, Brukiene V, Aleksejuniene J. Hemophilia and oral health. Stomatologija 2014; 16(4): 127-31.

2. Srivastava A, Brewer AK, Mauser Bunschoten EP et al. Treatment Guidelines Working Group. The World Federation of Hemophilia. Guidelines for the management of hemophilia. Haemophilia 2013; 19 : e1V14.

3. Gupta A, Epstein J B, Cabay RJ. Bleeding disorders of importance in dental care and related patient management. J Can Dent Assoc 2007; 73(1): 77-83

4. Nickles K, Wohlfeil M, Alesci S, et al. Comprehensive treatment of periodontitis in patients with von Willebrand disease. J Periodontol 2010; 81(10): 1432-40.

5. Durham TM, Hodges ED, Harper J, et al. Management of traumatic oralfacial injury in the hemophiliac patient with inhibitor: case report. Pediatr Dent 1993; 15(4): 282-7.

6. Hidaka N, Maeda K, Kawakami C, et al. Fibrinolytic activity in periodontal disease. The relationship between fibrinolytic activity and severity of periodontal disease. J Periodontol 1981; 52(4): 181-6.

7. Kumar JN, Kumar RA, Varadarajan R, Sharma N. Specialty dentistry for the hemophiliac: is there a protocol in place? Indian J Dent Res 2007; 18(2): 48-54

8. Anderson JA, Brewer A, Creagh D, Hook, et al. Guidance on the dental management of patients with haemophilia and congenital bleeding disorders. Br Dent J 2013; 215(10): 497-504.

9. Gornitsky M, Hammouda W, Rosen H. Rehabilitation of a hemophiliac with implants: a medical perspective and case report. J Oral Maxillofac Surg 2005; 63(5): 592-7.

10. Franchini M, Rossetti G, Tagliaferri A, et al. Dental procedures in adult patients with hereditary bleeding disorders: 10 years experience in three Italian Hemophilia Centers. Haemophilia 2005; 11(5): 504-9.

11. Vassilopoulos $P$, Palcanis K. Bleeding disorders and periodontology. Periodontol 2000 2007; 44: 211-23.

12. Lockhart PB, Gibson J, Pond SH, Leitch J. Dental management considerations for the patient with an acquired coagulopathy. Part 2: Coagulopathies from drugs. Br Dent J 2003; 195(9): 495-501.

13. Hay CR, Laurian $Y$, Verroust F, Preston FE, Kernoff PB. Induction of immune tolerance in patients with hemophilia $A$ and inhibitors treated with porcine VIIIC by home therapy. Blood 1990; 76(5): 882-6.

14. Gruppo RA, Valdez LP, Stout RD. Induction of immune tolerance in patients with hemophilia A and inhibitors. Am J Pediatr Hematol Oncol 1992; 14(1): 82-7.

15. Mariani G, Kroner B. Immune Tolerance Study Group (ITSG). Immune tolerance in hemophilia with factor VIII inhibitors: predictors of success. Haematologica 2001; 86(11): 1186-93.

\section{The Journal of} Haemophilia

\section{Practice}

\section{An open-access} journal for

sharing

experience in

the care of

people with

bleeding

disorders

www.haemjournal.com 